\title{
Quaternionic Dolbeault complex and vanishing theorems on hyperkähler manifolds
}

\author{
Misha Verbitsky
}

\begin{abstract}
Let $(M, I, J, K)$ be a compact hyperkähler manifold, $\operatorname{dim}_{\mathbb{H}} M=n$, and $L$ a non-trivial holomorphic line bundle on $(M, I)$. Using the quaternionic Dolbeault complex, we prove the following vanishing theorem for holomorphic cohomology of $L$. If $c_{1}(L)$ lies in the closure $\hat{K}$ of the dual Kähler cone, then $H^{i}(L)=0$ for $i>n$. If $c_{1}(L)$ lies in the opposite cone $-\hat{K}$, then $H^{i}(L)=0$ for $i<n$. Finally, if $c_{1}(L)$ is neither in $\hat{K}$ nor in $-\hat{K}$, then $H^{i}(L)=0$ for $i \neq n$.
\end{abstract}

\section{Introduction}

\subsection{Hypercomplex and hyperkähler manifolds}

Definition 1.1. Let $M$ be a manifold, and let $I, J, K \in \operatorname{End}(T M)$ be endomorphisms of the tangent bundle satisfying the quaternionic relation

$$
I^{2}=J^{2}=K^{2}=I J K=-\operatorname{Id}_{T M} .
$$

The manifold $(M, I, J, K)$ is called hypercomplex if the almost complex structures $I, J, K$ are integrable. If, in addition, $M$ is equipped with a Riemannian metric $g$ which is Kähler with respect to $I, J, K$, the manifold $(M, I, J, K, g)$ is called hyperkähler.

Consider the Kähler forms $\omega_{I}, \omega_{J}, \omega_{K}$ on $M$ :

$$
\omega_{I}(\cdot, \cdot):=g(\cdot, I \cdot), \quad \omega_{J}(\cdot, \cdot):=g(\cdot, J \cdot), \quad \omega_{K}(\cdot, \cdot):=g(\cdot, K \cdot) .
$$

An elementary linear-algebraic calculation implies that the 2 -form $\Omega:=\omega_{J}+\sqrt{-1} \omega_{K}$ is of Hodge type $(2,0)$ on $(M, I)$. This form is clearly closed and non-degenerate, hence it is a holomorphic symplectic form.

In algebraic geometry, the word 'hyperkähler' is essentially synonymous with 'holomorphically symplectic', due to the following theorem, which is implied by Yau's solution of the Calabi conjecture.

Theorem 1.2. Let $(M, I)$ be a compact, Kähler, holomorphically symplectic manifold. Then there exists a unique hyperkähler metric on $(M, I)$ with the same Kähler class.

Proof. For the proof, see [Yau78] and [Bes87].

Remark 1.3. The hyperkähler metric is unique, but there could be several hyperkähler structures compatible with a given hyperkähler metric on $(M, I)$.

Received 19 May 2006, accepted in final form 8 November 2006, published online 1 October 2007.

2000 Mathematics Subject Classification 53C26.

Keywords: hyperkähler, quaternion, holomorphic, cohomology vanishing.

The author is supported by EPSRC grant GR/R77773/01.

This journal is (C) Foundation Compositio Mathematica 2007. 


\section{VANISHING THEOREMS FOR HYPERKÄHLER MANIFOLDS}

Definition 1.4. Let $(M, I, J, K)$ be a compact, simply connected hyperkähler ${ }^{1}$ manifold which satisfies $H^{1}(M)=0, H^{2,0}(M, I)=\mathbb{C}$. Then $M$ is called an irreducible hyperkähler manifold.

Remark 1.5. By Bogomolov's decomposition theorem [Bog74], any compact holomorphic symplectic manifold admits a finite cover which is a product of several irreducible hyperkähler manifolds and a compact torus. This explains the term.

\subsection{Vanishing theorems on hyperkähler manifolds}

Using the argument which essentially belongs to the theory of hypercomplex manifolds, we are able to prove the following algebro-geometric statements.

Theorem 1.6. Let $(M, I, J, K)$ be a compact, irreducible hyperkähler manifold, and $L$ a holomorphic line bundle on $(M, I)$ with $c_{1}(L) \neq 0$. Denote by

$$
\overline{\mathcal{K}}^{\smile} \subset H^{1,1}(M, I) \cap H^{2}(M, \mathbb{R})
$$

the closure of the dual Kähler cone of $(M, I)(\S 5.2)$, and let $-\overline{\mathcal{K}}^{`}$ be the opposite cone. Then one of the following holds:

(i) $c_{1}(L) \in \overline{\mathcal{K}}^{\sim}$; then $H^{i}(L)=0$ for $i>\frac{1}{2} \operatorname{dim}_{\mathbb{C}} M$;

(ii) $c_{1}(L) \in-\overline{\mathcal{K}}^{\sim}$; then $H^{i}(L)=0$ for $i<\frac{1}{2} \operatorname{dim}_{\mathbb{C}} M$;

(iii) $c_{1}(L)$ does not lie in $-\overline{\mathcal{K}}^{\sim} \cup \overline{\mathcal{K}}^{\sim}$; then $H^{i}(L)=0$ for $i \neq \frac{1}{2} \operatorname{dim}_{\mathbb{C}} M$.

Proof. See Theorem 5.6.

TheOREm 1.7. Let $(M, I, J, K)$ be a compact, irreducible hyperkähler manifold, $L$ a holomorphic line bundle on $(M, I)$ with $c_{1}(L) \neq 0$, and $B$ an arbitrary holomorphic vector bundle on $(M, I)$. Then there exists a sufficiently big number $N_{0}$, such that for any integer $N>N_{0}$ one of the following holds:

(i) $c_{1}(L) \in \overline{\mathcal{K}}^{\sim}$; then $H^{i}\left(L^{N} \otimes B\right)=0$ for $i>\frac{1}{2} \operatorname{dim}_{\mathbb{C}} M$;

(ii) $c_{1}(L) \in-\overline{\mathcal{K}}^{\sim}$; then $H^{i}\left(L^{N} \otimes B\right)=0$ for $i<\frac{1}{2} \operatorname{dim}_{\mathbb{C}} M$;

(iii) $c_{1}(L)$ does not lie in $-\overline{\mathcal{K}}^{\sim} \cup \overline{\mathcal{K}}^{\sim}$; then $H^{i}\left(L^{N} \otimes B\right)=0$ for $i \neq \frac{1}{2} \operatorname{dim}_{\mathbb{C}} M$.

Proof. This is Theorem 5.8.

The vanishing theorems have many interesting geometrical consequences. As an example, we give the following theorem $(\S 6)$.

Theorem 1.8. Let $(M, I, J, K)$ be an irreducible hyperkähler manifold, and $X \subset(M, I)$ a subvariety of dimension $\operatorname{dim}_{\mathbb{C}} X>\frac{1}{2} \operatorname{dim}_{\mathbb{C}} M$. Assume that $X$ is a complete intersection of ample divisors. Consider a holomorphic line bundle $L$ on $(M, I)$ with $c_{1}(L)$ numerically effective (that is, $c_{1}(L)$ lies in the closure of the Kähler cone of $(M, I)$ ) and $q\left(c_{1}(L), c_{1}(L)\right)=0$, where $q$ is the BogomolovBeauville-Fujiki bilinear form (Definition 4.4). Then the natural restriction map is surjective on holomorphic sections,

$$
H^{0}\left(L^{N}\right) \rightarrow H^{0}\left(\left.L^{N}\right|_{X}\right) \rightarrow 0
$$

for a sufficiently big power of $L$.

Proof. See Theorem 6.5.

${ }^{1}$ Or holomorphically symplectic Kähler; by Yau's theorem, it is essentially the same notion. 


\section{VERBITSKY}

\section{Quaternionic Dolbeault complex}

\subsection{Weights of SU(2)-representations}

It is well known that any finite-dimensional (continuous) irreducible representations of $\mathrm{SU}(2)$ over $\mathbb{C}$ can be obtained as a symmetric power $S^{i}\left(V_{1}\right)$, where $V_{1}$ is a fundamental two-dimensional representation. We say that a representation $W$ has weight $i$ if it is isomorphic to $S^{i}\left(V_{1}\right)$. A representation is said to be pure of weight $i$ if all its irreducible components have weight $i$. If all irreducible components of a representation $W_{1}$ have weight at most $i$, we say that $W_{1}$ is a representation of weight at most $i$. In a similar fashion one defines representations of weight at least $i$.

Remark 2.1. The Clebsch-Gordan formula (see [Hum72]) claims that the weight is multiplicative, in the following sense: if $i \leqslant j$, then

$$
V_{i} \otimes V_{j}=\bigoplus_{k=0}^{i} V_{i+j-2 k},
$$

where $V_{i}=S^{i}\left(V_{1}\right)$ denotes the irreducible representation of weight $i$.

A subspace $W \subset W_{1}$ is pure of weight $i$ if the $\mathrm{SU}(2)$-representation $W^{\prime} \subset W_{1}$ generated by $W$ is pure of weight $i$.

\subsection{Quaternionic Dolbeault complex: a definition}

Let $M$ be a hypercomplex (e.g. a hyperkähler) manifold, $\operatorname{dim}_{\mathbb{H}} M=n$. There is a natural multiplicative action of $\mathrm{SU}(2) \subset \mathbb{H}^{*}$ on $\Lambda^{*}(M)$, associated with the hypercomplex structure.

The space $\Lambda^{*}(M)$ of differential forms is an infinite-dimensional representation of $\mathrm{SU}(2)$. However, the $\mathrm{SU}(2)$-action is $C^{\infty}(M)$-linear, because the group $\mathrm{SU}(2)$ acts on $\Lambda^{*}(M)$ fiberwise. Take a differential form $\gamma \in \Lambda^{*}(M)$ such that the restriction $\left.\gamma\right|_{x}$ of $\gamma$ to any fiber $\left.\Lambda^{*}(M)\right|_{x}$ lies in an irreducible, weight $k$ representation of $\mathrm{SU}(2)$ (this is possible, because the fiber $\left.\Lambda^{*}(M)\right|_{x}$ is finite-dimensional). Then $\gamma$ lies in a direct sum of weight $k$ representations. If we pick $\gamma$ in such a way that $\left.\gamma\right|_{x}$ is a vector of highest (or lowest) weight with respect to some Cartan subalgebra in $\mathfrak{s u}(2)$, then $\gamma$ is also a vector of highest (or lowest) weight, and generates an irreducible weight $k$ representation. It is easy to see that all irreducible subrepresentations of $\Lambda^{*}(M)$ are obtained this way. This gives the following claim.

Claim 2.2. All irreducible components of $\Lambda^{*}(M)$ are finite-dimensional.

Remark 2.3. From the above argument, we see that it makes sense to speak of the weight of $\Lambda^{*}(M)$ and its subrepresentations. Clearly, $\Lambda^{1}(M)$ has weight 1 . From the Clebsch-Gordan formula (Remark 2.1), it follows that $\Lambda^{i}(M)$ is an SU(2)-representation of weight at most $i$. Using the Hodge *-isomorphism $\Lambda^{i}(M) \cong \Lambda^{4 n-i}(M)$, we find that, for $i>2 n, \Lambda^{i}(M)$ is a representation of weight at most $2 n-i$.

Let $V^{i} \subset \Lambda^{i}(M)$ be a maximal $\mathrm{SU}(2)$-invariant subspace of weight less than $i$. The space $V^{i}$ is well defined, because it is a sum of all irreducible representations $W \subset \Lambda^{i}(M)$ of weight less than $i$. Since the weight is multiplicative (Remark 2.1), $V^{*}=\bigoplus_{i} V^{i}$ is an ideal in $\Lambda^{*}(M)$. We also have $V^{i}=\Lambda^{i}(M)$ for $i>2 n$ (Remark 2.3).

It is easy to see that the de Rham differential $d$ increases the weight by 1 at most. Therefore, $d V^{i} \subset V^{i+1}$, and $V^{*} \subset \Lambda^{*}(M)$ is a differential ideal in the de Rham DG-algebra $\left(\Lambda^{*}(M), d\right)$.

Definition 2.4 [Ver02]. Denote by $\left(\Lambda_{+}^{*}(M), d_{+}\right)$the quotient algebra $\Lambda^{*}(M) / V^{*}$. It is called the quaternionic Dolbeault algebra of $M$, or the quaternionic Dolbeault complex (qD-algebra or qDcomplex for short). 


\section{VANISHING THEOREMS FOR HYPERKÄHLER MANIFOLDS}

The space $\Lambda_{+}^{i}(M)$ can be identified with the maximal subspace of $\Lambda^{i}(M)$ of weight $i$, that is, a sum of all irreducible subrepresentations of weight $i$. This way, $\Lambda_{+}^{i}(M)$ can be considered as a subspace in $\Lambda^{i}(M)$; however, this subspace is not preserved by the multiplicative structure and the differential.

Remark 2.5. The complex $\left(\Lambda_{+}^{*}(M), d_{+}\right)$was constructed much earlier by Salamon [Sal07], in a different (and much more general) situation, and has been much studied since then [CS88, Bas92, LY97].

\subsection{The Hodge decomposition on the quaternionic Dolbeault complex}

Let $(M, I, J, K)$ be a hypercomplex manifold, and $L$ a complex structure induced by the quaternionic action, say, $I, J$ or $K$. Consider the $U(1)$-action on $\Lambda^{1}(M)$ provided by $\varphi \stackrel{\rho_{L}}{\longrightarrow} \cos \varphi \operatorname{Id}+\sin \varphi \cdot L$. We extend this action to a multiplicative action on $\Lambda^{*}(M)$. Clearly, for a $(p, q)$-form $\eta \in \Lambda^{p, q}(M, L)$, we have

$$
\rho_{L}(\varphi) \eta=e^{\sqrt{-1}(p-q) \varphi} \eta
$$

Lemma 2.6. Let $(M, I, J, K)$ be a hypercomplex manifold and

$$
\rho_{I}, \rho_{J}, \rho_{K}
$$

the homomorphisms

$$
U(1) \rightarrow \operatorname{Aut}\left(\Lambda^{*}(M)\right)
$$

constructed above. Then $\rho_{I}, \rho_{J}, \rho_{K}$ generate the Lie group action

$$
\mathrm{SU}(2) \subset \operatorname{Aut}\left(\Lambda^{*}(M)\right)
$$

associated with the hypercomplex structure.

Proof. Lemma 2.6 is clear. Indeed, the action of $\mathrm{SU}(2)$ and $\rho_{I}, \rho_{J}, \rho_{K}$ are defined on $\Lambda^{*}(M)$ by multiplicativity, hence it suffices to check that $\rho_{I}, \rho_{J}, \rho_{K}$ generate the standard action of $\mathrm{SU}(2)$ on $\Lambda^{1}(M)$. On $\Lambda^{1}(M), \rho_{I}, \rho_{J}, \rho_{K}$ act as quaternions $\cos \varphi+\sin \varphi \cdot I, \cos \varphi+\sin \varphi \cdot J, \cos \varphi+\sin \varphi \cdot K$, and they generate the group of unitary quaternions.

From Lemma 2.6, it is clear that $\rho_{L}$ preserves components of weight $i$. We obtain that $V^{*}$ is preserved by $\rho_{L}$, hence $\rho_{L}$ acts on $\Lambda_{+}^{*}(M)$. Then, (2.1) gives a Hodge decomposition on $\Lambda_{+}^{*}(M)$ :

$$
\Lambda_{+}^{i}(M)=\bigoplus_{p+q=i} \Lambda_{+, L}^{p, q}(M) .
$$

The following result is implied immediately by the standard calculations from the theory of $\mathrm{SU}(2)$-representations.

Proposition 2.7. Let $(M, I, J, K)$ be a hypercomplex manifold and

$$
\Lambda_{+}^{i}(M)=\bigoplus_{p+q=i} \Lambda_{+, I}^{p, q}(M)
$$

the Hodge decomposition of $q D$-complex defined above. Then there is a natural isomorphism

$$
\Lambda_{+, I}^{p, q}(M) \cong \Lambda^{0, p+q}(M, I)
$$

Proof. The following lemma is clear.

Lemma 2.8. Let $(M, I, J, K)$ be a hypercomplex manifold, $\operatorname{dim}_{\mathbb{H}} M=n$, and $p$ an integer, $0 \leqslant p \leqslant 2 n$. Then $\Lambda^{0, p}(M, I) \subset \Lambda^{p}(M)$ is pure of weight $p$. 


\section{VERBITSKY}

Proof. Consider the operator $W_{I}: \Lambda^{*}(M) \rightarrow \Lambda^{*}(M)$ mapping a form $\eta \in \Lambda^{p, q}(M, I)$ to $\sqrt{-1}(p-q) \eta$. Clearly, $W_{I}$ acts as a generator of $\mathfrak{u}(1)$, with $\mathfrak{u}(1)$ associated to $\rho_{I}: U(1) \rightarrow \operatorname{End}\left(\Lambda^{*}(M)\right)$. By Lemma 2.6, $W_{I} \in \mathfrak{s u}(2)$, where the $\mathfrak{s u}(2)$-action on $\Lambda^{*}(M)$ is associated with the standard action of $\mathrm{SU}(2)$. Writing $\mathfrak{s u}(2)$ explicitly in terms of generators $W_{I}, W_{J}$ and $W_{K}$, we find that $W_{I}$ generates a Cartan subalgebra of $\mathfrak{s u}(2)$ (indeed, the corresponding Lie group is a maximal compact torus of $\mathrm{SU}(2))$. Since the Cartan algebra $\mathbb{C} \cdot W_{I}$ acts on $\Lambda^{p, 0}(M, I)$ with weight $p$, the space $\Lambda^{p, 0}(M, I)$ is of weight at least $p$. On the other hand, $\Lambda^{p}(M)$ is a representation of weight at most $p$ (Remark 2.3). Therefore, $\Lambda^{p, 0}(M, I)$ is pure of weight $p$.

Remark 2.9. This argument also implies that $\Lambda^{0, p}(M, I)$ coincides with $\Lambda_{+, I}^{0, p}(M) \subset \Lambda_{+}^{p}(M)$ (here we consider $\Lambda_{+}^{p}(M)$ as a maximal SU(2)-invariant subspace of weight $p$ in $\left.\Lambda^{p}(M)\right)$.

Now, Proposition 2.7 is implied by the general machinery of $\mathrm{SU}(2)$-representations. If $R$ is a finite-dimensional SU(2)-representation of weight at most $p$, the Cartan algebra action splits $R$ onto weight components $R=\bigoplus R_{i}, i=-p,-p+2, \ldots, p-2, p$ the weights of the root $\sqrt{-1} W_{I}$ acting on $R_{i}$ as a multiplication by $i$. Moreover, if $R$ is pure of weight $p$, then all spaces $R_{i}$ are naturally isomorphic, with isomorphism provided by the $\mathrm{SU}(2)$-action.

In the case $R=\Lambda_{+}^{p}(M)$, the decomposition $R=\bigoplus R_{i}$ is precisely the Hodge decomposition, hence the spaces $\Lambda_{+, I}^{p, q}(M)$ are naturally isomorphic for all $p, q \geqslant 0$ satisfying $p+q=i$. We have proved Proposition 2.7.

\subsection{The Hodge decomposition on qD-complex: an explicit construction}

The isomorphism (2.2) can be made explicit, and also multiplicative, in the following way. Let $\Re$ be an irreducible two-dimensional representation of $\mathrm{SU}(2)$. Clearly, any irreducible $\mathrm{SU}(2)$ representation of weight $p$ is isomorphic to $S^{p} \mathfrak{R}$ (the $p$ th symmetric power of $\mathfrak{R}$ ). Consider the root $\sqrt{-1} W_{I} \in \mathfrak{s u}(2)$, constructed in $\S 2.3$. The corresponding $\mathfrak{s l}(2)$-triple can be written as

$$
f=W_{J}+\sqrt{-1} W_{K}, \quad g=W_{J}-\sqrt{-1} W_{K}, \quad h=\sqrt{-1} W_{I} .
$$

Let $x, y$ be a basis in $\Re$, such that $h x=x, h y=-y, g x=y, f y=x$.

Consider a hypercomplex manifold $(M, I, J, K)$. The bundle

$$
\mathfrak{S}:=\bigoplus_{p} S^{p} \Re \otimes \Lambda^{0, p}(M, I)
$$

is equipped with a natural multiplicative structure (we assume that the elements of $S^{p} \Re$ and $\Lambda^{0, q}(M, I)$ commute). We define the following $\mathrm{SU}(2)$-action on $\mathfrak{S}: \mathrm{SU}(2)$ acts trivially on $\Lambda^{0, p}(M, I)$, and in a standard way on $S^{p} \Re$.

Consider an isomorphism $\Re \otimes \Lambda^{0,1}(M, I) \rightarrow \Lambda^{1}(M)$ mapping $x \otimes \eta$ to $J(\eta)$ and $y \otimes \eta$ to $\eta$. This map is clearly $\mathrm{SU}(2)$-invariant. Using the multiplicative structure on $\mathfrak{S}$, it can be extended to an $\mathrm{SU}(2)$-invariant algebra homomorphism

$$
\bigoplus_{p} S^{p} \Re \otimes \Lambda^{0, p}(M, I) \rightarrow \Lambda_{+}^{*}(M)
$$

Proposition 2.10. In these assumptions, (2.4) is an algebra isomorphism.

Proof. Let $\mathfrak{S}^{p} \subset \mathfrak{S}$ denote the grading $p$ component. Bijectivity of the map (2.4) is checked in the same way as one proves Proposition 2.7: the Hodge components of $\mathfrak{S}^{p}$ are all isomorphic, because $\mathfrak{S}^{p}$ is a pure representation of weight $p$, and the same is true for $\Lambda_{+}^{p}(M)$. Therefore, it suffices to prove that the restriction of (2.4) to one Hodge component, say, $y^{p} \Lambda^{0, p}(M, I)$, induces an isomorphism

$$
y^{p} \Lambda^{0, p}(M, I) \rightarrow \Lambda_{+, I}^{0, p}(M) .
$$

This is implied by the equality $\Lambda^{0, p}(M, I)=\Lambda_{+, I}^{0, p}(M)$ (Remark 2.9). 


\section{VANISHING THEOREMS FOR HYPERKÄHLER MANIFOLDS}

\subsection{The $\bar{\partial}_{J}$-operator}

Let $(M, I, J, K)$ be a hypercomplex manifold. We extend

$$
J: \Lambda^{1}(M) \rightarrow \Lambda^{1}(M)
$$

to $\Lambda^{*}(M)$ by multiplicativity. Recall that

$$
J\left(\Lambda^{p, q}(M, I)\right)=\Lambda^{q, p}(M, I),
$$

because $I$ and $J$ anticommute on $\Lambda^{1}(M)$. Denote by

$$
\bar{\partial}_{J}: \Lambda^{p, q}(M, I) \rightarrow \Lambda^{p, q+1}(M, I)
$$

the operator $J \circ \partial \circ J$, where $\partial: \Lambda^{p, q}(M, I) \rightarrow \Lambda^{p+1, q}(M, I)$ is the standard Dolbeault operator on $(M, I)$, that is, the $(1,0)$ part of the de Rham differential. Since $\partial^{2}=0$, we have $\bar{\partial}_{J}^{2}=0$. Since $I, J, K$ are integrable, the operators $d, d_{I}:=I \circ d \circ I, d_{J}:=J \circ d \circ J, d_{K}:=K \circ d \circ K$ pairwise anticommute. Therefore, $\bar{\partial}=\frac{1}{2}\left(d-\sqrt{-1} d_{I}\right)$ anticommutes with $\bar{\partial}_{J}=\frac{1}{2}\left(d_{J}-\sqrt{-1} d_{K}\right)$. Writing the supercommutator as $\{\cdot, \cdot\}$, we express this as

$$
\left\{\bar{\partial}_{J}, \bar{\partial}_{J}\right\}=0, \quad\left\{\bar{\partial}_{J}, \bar{\partial}\right\}=0
$$

\subsection{The $\bar{\partial}, \bar{\partial}_{J}$-bicomplex}

Consider the quaternionic Dolbeault complex $\left(\Lambda_{+}^{*}(M), d_{+}\right)$constructed in $\S 2.2$. Using the Hodge decomposition, we can represent this complex as

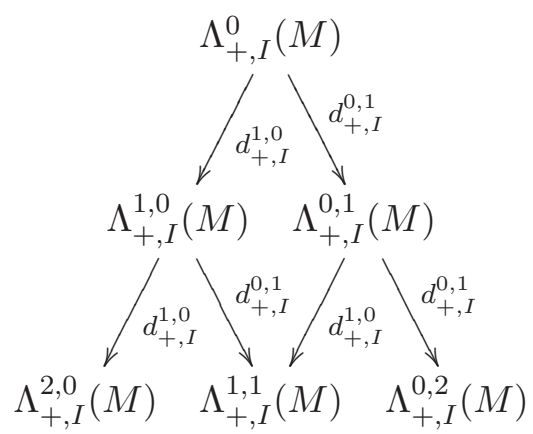

where $d_{+, I}^{1,0}$ and $d_{+, I}^{0,1}$ are the Hodge components of the quaternionic Dolbeault differential $d_{+}$, taken with respect to $I$.

Consider a hypercomplex manifold $(M, I, J, K)$. Let

$$
\bigoplus_{p} S^{p} \Re \otimes \Lambda^{0, p}(M, I) \rightarrow \Lambda_{+}^{*}(M)
$$

be the isomorphism constructed in Proposition 2.10. Writing the basis $x, y$ of $\mathfrak{R}$ as in the proof of Proposition 2.10, we may write the Hodge decomposition of (2.7) as

$$
x^{p} y^{q} \Lambda^{0, p+q}(M, I) \cong \Lambda_{+, I}^{p, q}(M) .
$$




\section{VERBITSKY}

Theorem 2.11. Under this correspondence, $d_{+}^{0,1}$ corresponds to $\bar{\partial}$ and $d_{+}^{1,0}$ to $\bar{\partial}_{J}$. This way the bicomplex (2.6) becomes equivalent to the bicomplex $\left(\Lambda^{0, p}(M, I), \bar{\partial}, \bar{\partial}_{J}\right)$ as follows:

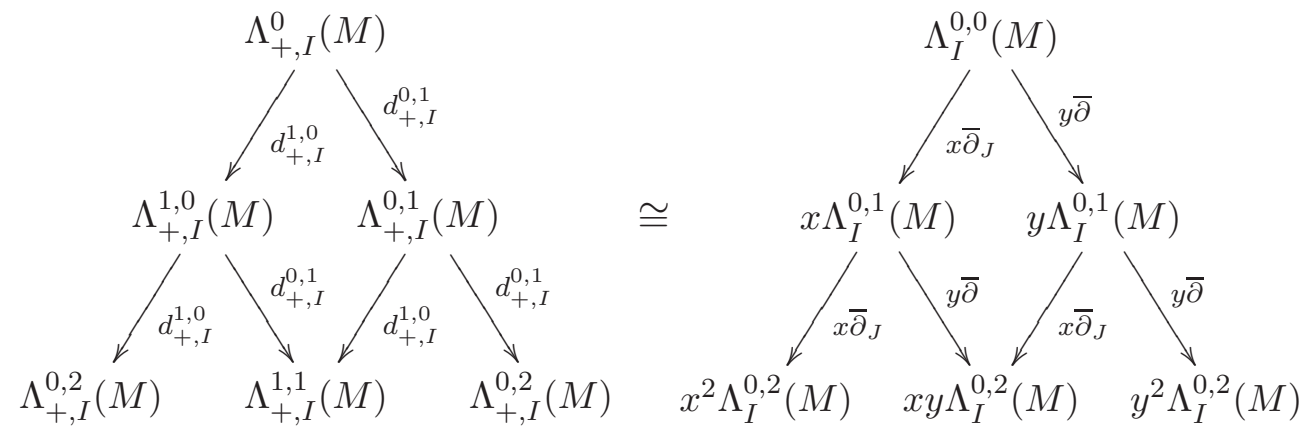

Proof. Consider the action of $x \bar{\partial}_{J}+y \bar{\partial}$ on

$$
\bigoplus_{p} S^{p} \Re \otimes \Lambda^{0, p}(M, I) \cong \Lambda_{+}^{*}(M)
$$

defined as in (2.8). To prove Theorem 2.11, we need to show that

$$
x \bar{\partial}_{J}+y \bar{\partial}=d_{+} \cdot
$$

Both of these operators satisfy the Leibniz rule, hence it suffices to check (2.9) on some set of multiplicative generators of $\Lambda_{+}^{*}(M)$. On $\Lambda_{+}^{0}(M)$, the equality (2.9) is clear from the definitions:

$$
x \bar{\partial}_{J}+\left.y \bar{\partial}\right|_{\Lambda_{+}^{0}(M)}=\left.d_{+}\right|_{\Lambda_{+}^{0}(M)} \cdot
$$

It is easy to check that the space $\Lambda^{0}(M) \oplus d \Lambda^{0}(M)$ generates the algebra $\Lambda^{*}(M)$. Therefore, $\Lambda_{+}^{0}(M) \oplus$ $d_{+} \Lambda_{+}^{0}(M)$ generates $\Lambda_{+}^{*}(M)$. To prove Theorem 2.11 it remains to show that

$$
x \bar{\partial}_{J}+\left.y \bar{\partial}\right|_{d_{+} \Lambda_{+}^{0}(M)}=\left.d_{+}\right|_{d_{+} \Lambda_{+}^{0}(M)} .
$$

Since $d_{+}^{2}=0$, we have $\left.d_{+}\right|_{d_{+} \Lambda_{+}^{0}(M)}=0$. By $(2.5)$, we have

$$
\left(x \bar{\partial}_{J}+y \bar{\partial}\right)^{2}=0 .
$$

Using (2.10) and (2.12), we obtain

$$
x \bar{\partial}_{J}+\left.y \bar{\partial}\right|_{d_{+} \Lambda_{+}^{0}(M)}=x \bar{\partial}_{J}+\left.y \bar{\partial}\right|_{x \bar{\partial}_{J}+y \bar{\partial}\left(\Lambda_{+}^{0}(M)\right)}=0 .
$$

Therefore, we have

$$
x \bar{\partial}_{J}+\left.y \bar{\partial}\right|_{d_{+} \Lambda_{+}^{0}(M)}=\left.d_{+}\right|_{d_{+} \Lambda_{+}^{0}(M)}=0 .
$$

This proves (2.11). Theorem 2.11 is proven.

\section{Kodaira identities for qD-complex}

\subsection{The Lefschetz-type $\mathfrak{s l}(2)$-action on $\Lambda^{0, *}(M, I) \otimes \operatorname{End}(B)$}

Let $(M, I, J, K)$ be a hyperkähler manifold, $B$ a holomorphic Hermitian vector bundle on $(M, I)$, and $\Lambda^{0, *}(M, I) \otimes B$ the space of $(0, p)$-forms with coefficients in $B$. Denote by $\bar{\Omega} \in \Lambda^{0,2}(M, I)$ the standard $(0,2)$-form $\omega_{J}+\sqrt{-1} \omega_{K}$ (see $\left.\S 1.1\right)$.

Using a hyperkähler metric, we construct a natural Hermitian structure on $\Lambda^{0, *}(M, I) \otimes B$. Denote by $L_{\bar{\Omega}}: \Lambda^{q, p}(M, I) \rightarrow \Lambda^{q, p+2}(M)$ the operator of exterior multiplication by $\bar{\Omega}$, and by $\Lambda_{\bar{\Omega}}: \Lambda^{q, p}(M, I) \otimes B \rightarrow \Lambda^{q, p-2}(M) \otimes B$ its Hermitian adjoint. The same argument as proves the usual Lefschetz theorem about the $\mathfrak{s l}(2)$-action (see [GH78]) can be used to prove the following linear-algebraic result, which is due to Fujiki [Fuj85]. 


\section{VANISHING THEOREMS FOR HYPERKÄHLER MANIFOLDS}

Proposition 3.1 [Fuj85]. With the above assumptions, let

$$
H_{\bar{\Omega}}:=\left[L_{\bar{\Omega}}, \Lambda_{\bar{\Omega}}\right]
$$

be a commutator of $L_{\bar{\Omega}}, \Lambda_{\bar{\Omega}}$. Then $H_{\bar{\Omega}}$ is a scalar operator, multiplying a $(q, p)$-form by $n-p$, where $n=\frac{1}{2} \operatorname{dim} \mathbb{H}(M)$. Moreover, $L_{\bar{\Omega}}, \Lambda_{\bar{\Omega}}, H_{\bar{\Omega}}$ is an $\mathfrak{s l}(2)$-triple.

Proof. For the proof, see [Ver96a, Theorem 4.2].

Let $\theta \in \Lambda^{0,1}(M, I) \otimes \operatorname{End}(B)$ be a 1-form. Denote by

$$
L_{\theta}: \Lambda^{q, p}(M, I) \otimes B \rightarrow \Lambda^{q, p+1}(M, I) \otimes B
$$

the operator of multiplication by $\theta$, and let

$$
\Lambda_{\theta}: \Lambda^{q, p}(M, I) \rightarrow \Lambda^{q, p-1}(M, I)
$$

be its Hermitian adjoint. Denote by $\theta_{J}$ the $(0,1)$-form $J(\bar{\theta})$.

Claim 3.2. In the above assumptions, we have

$$
\left[L_{\bar{\Omega}}, \Lambda_{\theta}\right]=L_{\theta_{J}}
$$

Proof. This follows from a trivial computation.

\section{$3.2 \bar{\partial}, \bar{\partial}_{J}$ with coefficients in a bundle}

Let $(M, I, J, K)$ be a hyperkähler manifold, and $B$ a holomorphic Hermitian vector bundle on $(M, I)$. Consider the standard (Chern) Hermitian connection $\nabla$ on $B, \nabla=\nabla^{1,0}+\bar{\partial}$, where $\bar{\partial}: B \rightarrow$ $B \otimes \Lambda^{0,1}(M, I)$ is the holomorphic structure operator. Denote by $\bar{\partial}_{J}: B \rightarrow B \otimes \Lambda^{0,1}(M, I)$ the composition of $\nabla^{1,0}: B \otimes \Lambda^{1,0}(M, I)$ and let

$$
\operatorname{Id}_{B} \otimes J: B \otimes \Lambda^{1,0}(M, I) \rightarrow B \otimes \Lambda^{0,1}(M, I)
$$

be an endomorphism associated with $J \in \mathbb{H}$. We extend $\bar{\partial}, \bar{\partial}_{J}$ to operators

$$
\bar{\partial}, \bar{\partial}_{J}: \Lambda^{0, p}(M, I) \otimes B \rightarrow \Lambda^{0, p+1}(M, I) \otimes B
$$

using the Leibniz rule.

Proposition 3.3. With these assumptions, $\bar{\partial}^{2}=\bar{\partial}_{J}^{2}=0$, and the anticommutator $\left\{\bar{\partial}, \bar{\partial}_{J}\right\}$ acts on $\Lambda^{0, *}(M, I)$ as a multiplication by an $\operatorname{End}(B)$-valued 2 -form $\Theta_{+} \in \Lambda^{0,2}(M, I) \otimes$ End $B$. Moreover, under the identification

$$
\Lambda^{0,2}(M, I) \otimes \text { End } B \cong \Lambda_{+, I}^{1,1}(M) \otimes \text { End } B
$$

(Proposition 2.7), $\Theta_{+}$corresponds to the $\Lambda_{+}^{2}(M)$ part of the curvature of $B$.

Proof. Let

$$
\nabla_{+}: \Lambda_{+}^{p}(M) \otimes B \rightarrow \Lambda_{+}^{p+1}(M) \otimes B
$$

be the connection operator restricted to $\Lambda_{+}^{*}(M) \otimes B$, and $\nabla_{+}=\nabla_{+}^{1,0}+\partial_{+}$its Hodge decomposition. Clearly, $\nabla_{+}^{2}$ is the $\Lambda_{+}^{2}(M)$ part of the curvature of $B$.

Now, Proposition 3.3 follows immediately from Theorem 2.11. Indeed, under the isomorphism (2.8), $x \bar{\partial}_{J}$ corresponds to $\nabla_{+}^{1,0}$; since the curvature of the Chern connection is of type $(1,1)$, we have $\left(\nabla_{+}^{1,0}\right)^{2}=0$, hence $\bar{\partial}_{J}^{2}=0$. Similarly, the operator $\left\{x \bar{\partial}_{J}, y \bar{\partial}\right\}$ under the isomorphism (2.8) corresponds to $\left\{\nabla_{+}^{1,0}, \partial_{+}\right\}=\nabla_{+}^{2}$. 


\section{VERBITSKY}

\subsection{Kodaira relations for $\bar{\partial}, \bar{\partial}_{J}$}

Let $(M, I, J, K)$ be a hyperkähler manifold. Consider the bicomplex

$$
\left(\Lambda^{0, *}(M, I), \bar{\partial}, \bar{\partial}_{J}\right),
$$

constructed in $\S 2.6$. Let

$$
L_{\bar{\Omega}}: \Lambda^{0, p}(M, I) \rightarrow \Lambda^{0, p+2}(M, I)
$$

be an operator of exterior multiplication by $\bar{\Omega}(\S 3.1)$, and

$$
\bar{\partial}^{*}, \bar{\partial}_{J}^{*}: \Lambda^{0, p}(M, I) \rightarrow \Lambda^{0, p-1}(M, I)
$$

the operators Hermitian adjoint to $\bar{\partial}, \bar{\partial}_{J}$.

The following proposition is well known.

Proposition 3.4. With these assumptions, the following commutator relations hold

$$
\left[L_{\bar{\Omega}}, \bar{\partial}^{*}\right]=\bar{\partial}_{J}, \quad\left[L_{\bar{\Omega}}, \bar{\partial}_{J}^{*}\right]=-\bar{\partial} .
$$

Proof. The proof of (3.2) is essentially the same as the proof of the usual Kodaira relations; see e.g. [Ver96a, Proposition 4.2].

The same argument, applied locally to $\operatorname{End}(B)$-valued forms, gives the following theorem.

Theorem 3.5. Let $(M, I, J, K)$ be a hyperkähler manifold, $B$ a holomorphic Hermitian vector bundle on $(M, I)$,

$$
\bar{\partial}, \bar{\partial}_{J}: \Lambda^{0, p}(M, I) \otimes B \rightarrow \Lambda^{0, p+1}(M, I) \otimes B
$$

the operators constructed in $\S 3.2$, and $\bar{\partial}^{*}, \bar{\partial}_{J}^{*}$ the Hermitian adjoint operators. Then

$$
\left[L_{\bar{\Omega}}, \bar{\partial}^{*}\right]=\bar{\partial}_{J}, \quad\left[L_{\bar{\Omega}}, \bar{\partial}_{J}^{*}\right]=-\bar{\partial} .
$$

Proof. For this, see [Ver96a].

\subsection{Kodaira-Nakano identities}

The following theorem is the qD-analogue of the usual Kodaira-Nakano identity (or, rather, the identity used in the proof of Kodaira-Nakano vanishing).

Theorem 3.6. Let $(M, I, J, K)$ be a hyperkähler manifold, $B$ a holomorphic Hermitian vector bundle on $(M, I)$,

$$
\bar{\partial}, \bar{\partial}_{J}: \Lambda^{0, p}(M, I) \otimes B \rightarrow \Lambda^{0, p+1}(M, I) \otimes B
$$

the operators constructed in $\S 3.2$, and $\bar{\partial}^{*}, \bar{\partial}_{J}^{*}$ the Hermitian adjoint operators. Consider the Laplacians

$$
\Delta_{\bar{\partial}}:=\left\{\bar{\partial}, \bar{\partial}^{*}\right\}, \quad \Delta_{\bar{\partial}_{J}}:=\left\{\bar{\partial}_{J}, \bar{\partial}_{J}^{*}\right\}
$$

(here, as elsewhere, $\{\cdot, \cdot\}$ denotes the anticommutator). Then

$$
\Delta_{\bar{\partial}}-\Delta_{\bar{\partial}_{J}}=\left[\Theta_{+}, \Lambda_{\bar{\Omega}}\right]
$$

where

$$
\Theta_{+}: \Lambda^{0, p}(M, I) \otimes B \rightarrow \Lambda^{0, p+2}(M, I)
$$

is an operator defined as

$$
\Theta_{+}:=\left\{\bar{\partial}, \bar{\partial}_{J}\right\}
$$

and identified with the $\Lambda_{+}^{2}(M) \otimes$ End $B$ part of the curvature of $B$ as in Proposition 3.3.

Proof. Using the graded Jacobi identity and Theorem 3.5, we obtain

$$
\left[\Theta_{+}, \Lambda_{\bar{\Omega}}\right]=-\left[\Lambda_{\bar{\Omega}},\left\{\bar{\partial}, \bar{\partial}_{J}\right\}\right]=\left\{\bar{\partial}, \bar{\partial}^{*}\right\}-\left\{\bar{\partial}_{J}, \bar{\partial}_{J}^{*}\right\}=\Delta_{\bar{\partial}}-\Delta_{\bar{\partial}_{J}} .
$$




\section{VANISHING THEOREMS FOR HYPERKÄHLER MANIFOLDS}

\section{Cohomology of hyperkähler manifolds}

For the convenience of the reader, we recall here some well-known facts about the structure of $H^{2}(M)$ for $M$ a compact, irreducible hyperkähler manifold; see [Bog78], [Bes87], [Bea83] and [Fuj85] for details.

\subsection{SU(2)-action on $H^{2}(M)$}

Let $(M, I, J, K, g)$ be a compact, irreducible hyperkähler manifold. Since $g$ is Kähler with respect to $(I, J, K)$, we have

$$
\nabla I=\nabla J=\nabla K=0
$$

where $\nabla$ denotes the Levi-Civita connection. Chern has shown that covariantly constant endomorphisms of $\Lambda^{*}(M)$ commute with the Laplacian (see [Bes87]). Then the $\mathrm{SU}(2)$-action generated by $I, J, K \in \mathbb{H}^{*}$ also commutes with the Laplacian. This gives an $\mathrm{SU}(2)$-action on the space of harmonic forms on $M$. Identifying the harmonic forms with cohomology, we obtain an $\mathrm{SU}(2)$-action on the cohomology as well.

Let $H^{2}(M)=H_{+}^{2}(M) \oplus H^{2}(M)_{\mathrm{SU}(2) \text {-inv }}$ be a decomposition of $H^{2}(M)$ onto its weight 2 and weight 0 components. Using the weights of the Cartan algebra action as in the proof of Proposition 2.7 , we find that

$$
\operatorname{dim} H^{2,0}(M, I)=\operatorname{dim} H_{+}^{1,1}(M, I)=\operatorname{dim} H^{0,2}(M, I) .
$$

Since $M$ is irreducible, $\operatorname{dim} H^{2,0}(M, I)=1$ and the space $H_{+}^{1,1}(M, I)$ is one-dimensional. Let $H^{2}(M)_{\mathrm{SU}(2) \text {-inv }}$ be the space of $\mathrm{SU}(2)$-invariant classes. It is easy to check that $\mathrm{SU}(2)$-invariant classes are all of type $(1,1)$ (e.g. [Ver96a]).

Since $H_{+}^{1,1}(M, I)$ is one-dimensional and generated by the Kähler form $\omega_{I}$, we have a decomposition

$$
H^{1,1}(M, I)=\mathbb{C} \omega_{I} \oplus H^{2}(M)_{\mathrm{SU}(2) \text {-inv }} .
$$

Using the $\mathfrak{s o}(1,4)$-action generated by the three Lefschetz $\mathfrak{s l}(2)$-triples associated with the Kähler structures $I, J, K$ as in [Ver90], we can easily show that an $\mathrm{SU}(2)$-invariant 2-form is primitive ${ }^{2}$ (see e.g. [Ver96a]).

This gives the following well-known statement [Ver96a].

Claim 4.1. Let $(M, I, J, K)$ be a compact, irreducible hyperkähler manifold. Then the space $H_{\text {prim }}^{1,1}(M, I)$ of primitive classes in $H^{1,1}(M, I)$ coincides with the space $H^{2}(M)_{\mathrm{SU}(2) \text {-inv }}$ of $\mathrm{SU}(2)$-invariant classes.

Proof. Since all SU(2)-invariant classes are primitive, $H_{\text {prim }}^{1,1}(M, I)$ contains $H^{2}(M)_{\mathrm{SU}(2) \text {-inv }}$. Comparing the decomposition (4.1) with

$$
H^{1,1}(M, I)=H_{\text {prim }}^{1,1}(M, I) \oplus \mathbb{C} \omega_{I},
$$

we find that $\operatorname{dim} H_{\text {prim }}^{1,1}(M, I)=\operatorname{dim} H^{2}(M)_{\mathrm{SU}(2) \text {-inv }}$.

\subsection{The Bogomolov-Beauville-Fujiki form}

Let $(M, I, J, K)$ be a compact hyperkähler manifold, and $\Omega:=\omega_{J}+\sqrt{-1} \omega_{K}$ the holomorphic symplectic form on $(M, I)$. Bogomolov [Bog78] defined the following bilinear symmetric 2-form

${ }^{2}$ Recall that the primitive classes [GH78] are cohomology classes which satisfy $\Lambda(\eta)=0$, where $\Lambda: H^{i}(M) \rightarrow H^{i-2}(M)$ is the dual Lefschetz operator. A $(1,1)$-class is primitive if and only if it is orthogonal to the Kähler form with respect to the Riemann-Hodge pairing. 


\section{VERBITSKY}

on $H^{1,1}(M, I)$ :

$$
\widetilde{q}\left(\eta, \eta^{\prime}\right):=\int_{M} \eta \wedge \eta^{\prime} \wedge \Omega^{n-1} \wedge \bar{\Omega}^{n-1},
$$

where $n=\operatorname{dim} \mathbb{H} M$. Since $\Omega \wedge \bar{\Omega}$ is a positive $(2,2)$-form, $\widetilde{q}$ is positive on the Kähler cone of $(M, I)$ :

$$
\forall \omega \in \mathcal{K}, \quad \widetilde{q}(\omega, \omega)>0 .
$$

An elementary linear-algebraic calculation similar to the proof of Riemann-Hodge bilinear relations implies that $\widetilde{q}(\eta, \eta)<0$ for $\eta$ primitive. Therefore, $\widetilde{q}$ has signature $(+,-,-,-, \ldots)$ on $H^{1,1}(M, I) \cap$ $H^{2}(M, \mathbb{R})$.

The form $\widetilde{q}$ is topological by its nature.

Theorem 4.2 [Fuj85]. Let $(M, I, J, K)$ be a compact, irreducible hyperkähler manifold of real dimension $4 n$. Then there exists a bilinear, symmetric non-degenerate 2-form $q: H^{2}(M, \mathbb{Q}) \otimes$ $H^{2}(M, \mathbb{Q}) \rightarrow \mathbb{Q}$ such that

$$
\int_{M} \eta^{2 n}=q(\eta, \eta)^{n}
$$

for all $\eta \in H^{2}(M)$. Moreover, $q$ is proportional to the form (4.2) on $H^{1,1}(M)$, and has signature $(+,+,+,-,-,-, \ldots)$.

Remark 4.3. If $n$ is odd, (4.4) determines $q$ uniquely, otherwise up to a sign. To choose a sign, we use (4.3).

Definition 4.4. Let $(M, I, J, K)$ be a compact, irreducible hyperkähler manifold. A BogomolovBeauville-Fujiki form on $M$ is a form

$$
q: H^{2}(M, \mathbb{Q}) \otimes H^{2}(M, \mathbb{Q}) \rightarrow \mathbb{Q}
$$

which satisfies (4.4), and takes positive values on the Kähler cone of $(M, I)$. Such a form always exists and is unique, by Theorem 4.2 .

Remark 4.5. The Bogomolov-Beauville-Fujiki form is integer, but not unimodular on $H^{2}(M, \mathbb{Z})$.

The Bogomolov-Beauville-Fujiki form can be expressed in terms of the SU(2)-action on cohomology $(\S 4.1)$ as follows.

Claim 4.6. Let $(M, I, J, K)$ be a compact, irreducible hyperkähler manifold, and $(\cdot, \cdot)_{\mathcal{H}}$ the positive definite pairing on cohomology associated with the Euclidean metric on the space of harmonic forms induced by the Riemannian structure. Consider the form $q^{\prime}$ which is equal to $(\cdot, \cdot)_{\mathcal{H}}$ on the threedimensional space generated by $\omega_{I}, \omega_{J}, \omega_{K}$, and to $-(\cdot, \cdot)_{\mathcal{H}}$ on its orthogonal complement. Then $q^{\prime}$ is proportional to the Bogomolov-Beauville-Fujiki form.

Proof. For the proof, see e.g. [Ver96b, Theorem 2.1].

This immediately gives the following corollary.

Corollary 4.7. Consider the natural SU(2)-action on the cohomology of a hyperkähler manifold. Then the Bogomolov-Beauville-Fujiki form is $\mathrm{SU}(2)$-invariant.

Using the Riemann-Hodge bilinear relations, we can express $(\cdot, \cdot)_{\mathcal{H}}$ in terms of the product structure on cohomology. Together with Claim 4.6, this gives

$$
q^{\prime}\left(\eta_{1}, \eta_{2}\right)=\int_{X} \omega_{I}^{2 n-2} \wedge \eta_{1} \wedge \eta_{2}-\frac{2 n-2}{(2 n-1)^{2}} \cdot \frac{\int_{X} \omega_{I}^{2 n-1} \eta_{1} \cdot \int_{X} \omega_{I}^{2 n-1} \eta_{2}}{\int_{X} \omega_{I}^{n}},
$$

for any $\eta_{1}, \eta_{2} \in H^{2}(M)$ (see [Ver95, Claim 5.1]). This formula is due to Beauville.

The following claim follows directly from (4.5) and Claim 4.1. 


\section{VANISHING THEOREMS FOR HYPERKÄHLER MANIFOLDS}

Claim 4.8. Let $(M, I, J, K)$ be an irreducible, compact hyperkähler manifold, and $\eta \in H^{1,1}(M, I)$ a $(1,1)$-class. Then the following assertions are equivalent:

(i) $q\left(\eta, \omega_{I}\right)=0$, where $q$ is the Bogomolov-Beauville-Fujiki form, and $\omega_{I}$ the Kähler class of $(M, I)$;

(ii) $\eta$ is primitive;

(iii) $\eta$ is $\mathrm{SU}(2)$-invariant.

Proof. The equivalence of assertions (ii) and (iii) is implied by Claim 4.1, and the equivalence of assertions (i) and (iii) by (4.5).

\section{The vanishing of cohomology}

\subsection{Cohomology vanishing for line bundles with $q\left(c_{1}(L), \omega\right)>0$}

The following result is implied immediately by the quaternionic Kodaira-Nakano identity (Theorem 3.6), in the same fashion as the usual Kodaira-Nakano vanishing follows from the usual Kodaira-Nakano identity.

Proposition 5.1. Let $(M, I, J, K)$ be a compact, irreducible hyperkähler manifold, $\operatorname{dim}_{\mathbb{R}} M=4 n$, and $L$ a holomorphic line bundle on $(M, I)$, such that $q\left(c_{1}(L), \omega_{I}\right)>0$, where $\omega_{I}$ is the Kähler class of $(M, I)$. Then the holomorphic cohomology $H^{i}(M, L)$ is zero for $i>n$.

Proof. Let $\eta$ be a harmonic form representing $c_{1}(L)$. We may chose the Hermitian structure on $L$ in such a way that $\eta$ is equal to the curvature of $L$ (see [GH78]). Let $\omega$ denote the Kähler form of $(M, I)$. Abusing the notation, we denote the Kähler class of $(M, I)$ by the same letter.

The cohomology class

$$
\kappa:=c_{1}(L)-\frac{q\left(c_{1}(L), \omega\right)}{q(\omega, \omega)} \omega
$$

clearly satisfies $q(\kappa, \omega)=0$. Therefore, $\kappa$ is $\mathrm{SU}(2)$-invariant (Claim 4.8). Since $\eta$ is harmonic, the harmonic form

$$
\eta-\frac{q\left(c_{1}(L), \omega\right)}{q(\omega, \omega)} \omega
$$

representing $\kappa$ is also $\mathrm{SU}(2)$-invariant. Let $\widetilde{\omega}$ be the form

$$
\omega \in \Lambda_{+, I}^{1,1}(M)
$$

considered as an element in $\Lambda^{0,2}(M, I)$ using the isomorphism constructed in Proposition 2.7. By Proposition 3.3,

$$
\Theta_{+}=\left\{\bar{\partial}, \bar{\partial}_{J}\right\}=\frac{q\left(c_{1}(L), \omega\right)}{q(\omega, \omega)} \widetilde{\omega} .
$$

Clearly, $\omega_{I}, \omega_{J}, \omega_{K}$ form a three-dimensional irreducible $\mathrm{SU}(2)$-invariant subspace of $\Lambda^{2}(M)$. A trivial calculation is used to show that $\widetilde{\omega}$ is in fact equal to $\bar{\Omega}$. This gives

$$
\Theta_{+}=\left\{\bar{\partial}_{,} \bar{\partial}_{J}\right\}=\lambda L_{\bar{\Omega}},
$$

where $\lambda=q\left(c_{1}(L), \omega\right) / q(\omega, \omega)$ is a positive constant. Comparing (5.3), Kodaira-Nakano identity (3.4) and the quaternionic Lefschetz theorem (Proposition 3.1), we obtain

$$
\Delta_{\bar{\partial}}-\Delta_{\bar{\partial}_{J}}=\left[\Theta_{+}, \Lambda_{\bar{\Omega}}\right]=\lambda H_{\bar{\Omega}}
$$

On $(0, i)$-forms this operator acts as $(i-n) \lambda$. Given a harmonic form

$$
\nu \in \operatorname{ker} \Delta_{\bar{\partial}} \subset \Lambda^{0, i}(M, I) \otimes L,
$$




\section{VERBITSKY}

we can obtain

$$
0=\Delta_{\bar{\partial}}(\nu)=\Delta_{\bar{\partial}_{J}}(\nu)+(i-n) \lambda \nu
$$

Since

$$
\left(\Delta_{\bar{\partial}_{J}}(\nu), \nu\right)=\left(\bar{\partial}_{J} \eta, \bar{\partial}_{J} \eta\right)+\left(\bar{\partial}_{J}^{*} \eta, \bar{\partial}_{J}^{*} \eta\right) \geqslant 0,
$$

(5.5) leads to $(\nu, \nu)=0$ for $i>n$. The harmonic $(0, i)$-forms are identified with the $i$ th holomorphic cohomology of $L$ as usual. We have proved Proposition 5.1.

Remark 5.2. Let $W$ be a Hermitian vector space. A positive operator $A: W \rightarrow W$ is an operator which satisfies $(A(x), x) \geqslant 0$ for all $x \in W$. Operator $A$ is positive definite if this inequality is strict for all non-zero $x$. From (5.6), we obtain that the Laplacians $\Delta_{\bar{\partial}_{J}}$ and $\Delta_{\bar{\partial}}$ are positive. If

$$
\Delta_{\bar{\partial}}=\Delta_{\bar{\partial}_{J}}+A
$$

where $A$ is positive definite, then ker $\Delta_{\bar{\partial}}=0$. This argument is used quite often in geometry and analysis.

Remark 5.3. Let $(M, I, J, K)$ be a compact, irreducible hyperkähler manifold, $\operatorname{dim}_{\mathbb{H}} M=n$, and $L$ a holomorphic line bundle on $(M, I)$. Then Serre's duality gives $H^{i}(M, L)^{*} \cong H^{n-i}\left(M, L^{*}\right)$, because the canonical class of $M$ is trivial. Therefore, Proposition 5.1 implies that $H^{i}(M, L)$ vanishes for all $i<n$ if $L$ is a holomorphic line bundle on $(M, I)$ with $q\left(c_{1}(L), \omega\right)<0$.

\subsection{The dual Kähler cone and vanishing}

Let $(M, I)$ be a Kähler manifold.

Definition 5.4. The Kähler cone $\mathcal{K} \subset H^{1,1}(M, I)$ for $(M, I)$ is the set of all Kähler classes $\omega \in$ $H_{I}^{1,1}(M, \mathbb{R})$, where $H_{I}^{1,1}(M, \mathbb{R})$ denotes the intersection $H^{1,1}(M, I) \cap H^{2}(M, \mathbb{R})$. Clearly, $\mathcal{K}$ is a convex cone in $H_{I}^{1,1}(M, \mathbb{R})$.

Now, let $(M, I, J, K)$ be a hyperkähler manifold, $\mathcal{K} \subset H_{I}^{1,1}(M, \mathbb{R})$ the Kähler cone of $(M, I)$, and $q: H_{I}^{1,1}(M, \mathbb{R}) \times H_{I}^{1,1}(M, \mathbb{R}) \rightarrow \mathbb{R}$ the Bogomolov-Beauville-Fujiki form. We define the dual Kähler cone

$$
\mathcal{K}^{\sim} \subset H_{I}^{1,1}(M, \mathbb{R})
$$

as

$$
\mathcal{K}^{\sim}:=\left\{x \in H_{I}^{1,1}(M, \mathbb{R}) \mid \forall y \in \mathcal{K}, \quad q(x, y)>0\right\} .
$$

It is an open, convex cone. Since a product of two Kähler forms is positive, we have $\mathcal{K}^{\sim} \supset \mathcal{K}$.

Denote by $\overline{\mathcal{K}}^{\curvearrowright}$ the closure of $\mathcal{K}^{\sim}$ in $H_{I}^{1,1}(M, \mathbb{R})$, and by $-\overline{\mathcal{K}}^{\curvearrowright}$ the opposite cone. Clearly,

$$
\overline{\mathcal{K}}^{\sim}:=\left\{x \in H_{I}^{1,1}(M, \mathbb{R}) \mid \forall y \in \mathcal{K}, \quad q(x, y) \geqslant 0\right\}
$$

and

$$
-\overline{\mathcal{K}}^{\vee}:=\left\{x \in H_{I}^{1,1}(M, \mathbb{R}) \mid \forall y \in \mathcal{K}, \quad q(x, y) \leqslant 0\right\} .
$$

Proposition 5.1 immediately leads to the following corollary.

Corollary 5.5. Let $(M, I)$ be a compact, irreducible, holomorphically symplectic Kähler manifold, $\operatorname{dim}_{\mathbb{C}} M=2 n$, and $L$ a holomorphic line bundle on $(M, I)$ with $c_{1}(L) \notin \overline{\mathcal{K}}$. Then the holomorphic cohomology $H^{i}(M, L)$ is zero for all $i<n$.

Now we can prove the main result of this paper.

Theorem 5.6. Let $(M, I, J, K)$ be a compact, irreducible hyperkähler manifold, and $L$ a holomorphic line bundle on $(M, I)$ with $c_{1}(L) \neq 0$. Then one of the following holds: 


\section{VANISHING THEOREMS FOR HYPERKÄHLER MANIFOLDS}

(i) $c_{1}(L) \in \overline{\mathcal{K}}^{\sim}$; then $H^{i}(L)=0$ for $i>\frac{1}{2} \operatorname{dim}_{\mathbb{C}} M$;

(ii) $c_{1}(L) \in-\overline{\mathcal{K}}^{\sim}$; then $H^{i}(L)=0$ for $i<\frac{1}{2} \operatorname{dim}_{\mathbb{C}} M$;

(iii) $c_{1}(L)$ does not lie in $-\overline{\mathcal{K}}^{\sim} \cup \overline{\mathcal{K}}^{\sim}$; then $H^{i}(L)=0$ for $i \neq \frac{1}{2} \operatorname{dim}_{\mathbb{C}} M$.

Proof. Denote $\frac{1}{2} \operatorname{dim}_{\mathbb{C}} M$ by $n$. Theorem 5.6(iii) is a direct consequence of Corollary 5.5. Indeed, in this case

$$
H^{i}(L)=0 \text { for } i<n
$$

and

$$
H^{i}\left(L^{*}\right)=0 \text { for } i<n,
$$

because the Chern classes of both $L$ and $L^{*}$ do not lie in $\overline{\mathcal{K}}$. However, by Serre's duality, (5.7) is equivalent to

$$
H^{i}(L)=0 \quad \text { for } i>n .
$$

Let us prove Theorem 5.6(i). Since $c_{1}(L) \in \overline{\mathcal{K}}^{\sim}$, we may assume that $q\left(c_{1}(L), \omega\right) \geqslant 0$ for all $\omega \in \mathcal{K}$. Unless $q\left(c_{1}(L), \omega\right)=0$ for all Kähler classes $\omega$, the assertion of Theorem 5.6(i) is obtained from Proposition 5.1. However, if $q\left(c_{1}(L), \omega\right)=0$ for all Kähler classes, $c_{1}(L)=0$, because $q$ is non-degenerate and the Kähler classes generate $H_{I}^{1,1}(M, \mathbb{R})$. Theorem 5.6(i) is obtained from (ii) by Serre's duality.

The classes $\eta \notin-\overline{\mathcal{K}}^{\sim} \cup \overline{\mathcal{K}}^{`}$ can also be characterized as follows.

Claim 5.7. Let $(M, I, J, K)$ be a compact, irreducible hyperkähler manifold, and $\eta \in H_{I}^{1,1}(M, \mathbb{R})$ a non-zero cohomology class. Then the following conditions are clearly equivalent:

(i) $\eta \notin-\overline{\mathcal{K}^{\sim}} \cup \overline{\mathcal{K}^{2}}$;

(ii) $q\left(\eta, \omega_{1}\right)>0$ and $q\left(\eta, \omega_{2}\right)<0$ for some Kähler forms $\omega_{1}, \omega_{2}$ on $(M, I)$;

(iii) $\eta$ is primitive with respect to some Kähler form on $(M, I)$; or, equivalently, $q(\eta, \omega)=0$ (see Claim 4.8);

(iv) the class $\eta$ is $\mathrm{SU}(2)$-invariant with respect to some hyperkähler structure $\left(I, J^{\prime}, K^{\prime}\right)$ on $M$.

Proof. The equivalence of conditions (i) and (ii) is clear. The equivalence of conditions (iii) and (iv) is implied by Claim 4.8. The implication (ii) $\Rightarrow$ (iii) is clear, because the Kähler cone is connected, hence from $q\left(\eta, \omega_{1}\right)>0$ and $q\left(\eta, \omega_{2}\right)<0$ it follows that $q\left(\eta, \omega_{3}\right)=0$ for some Kähler form. Finally, (iii) $\Rightarrow$ (ii) is obtained as follows: Given a Kähler class $\omega$, with $q(\eta, \omega)=0$, take a neighbourhood $U$ of $\omega$ in the Kähler cone. The function $U \stackrel{v}{\rightarrow} \mathbb{R}, v\left(\omega^{\prime}\right)=q\left(\eta, \omega^{\prime}\right)$, is non-zero and linear, hence it takes positive and negative values in any open neighbourhood of $\omega$.

\subsection{Cohomology vanishing for vector bundles of arbitrary rank}

A version of Theorem 5.6 can be stated for holomorphic bundles of arbitrary rank, as follows.

TheOrem 5.8. Let $(M, I, J, K)$ be a compact, irreducible hyperkähler manifold, $L$ a holomorphic line bundle on $(M, I)$ with $c_{1}(L) \neq 0$, and $B$ an arbitrary holomorphic vector bundle on $(M, I)$. Then there exists a sufficiently big number $N_{0}$, such that for any integer $N>N_{0}$ one of the following holds:

(i) $c_{1}(L) \in \overline{\mathcal{K}}^{\sim}$; then $H^{i}\left(L^{N} \otimes B\right)=0$ for $i>\frac{1}{2} \operatorname{dim}_{\mathbb{C}} M$;

(ii) $c_{1}(L) \in-\overline{\mathcal{K}}^{r}$; then $H^{i}\left(L^{N} \otimes B\right)=0$ for $i<\frac{1}{2} \operatorname{dim}_{\mathbb{C}} M$;

(iii) $c_{1}(L)$ does not lie in $-\overline{\mathcal{K}}^{\sim} \cup \overline{\mathcal{K}}^{\sim}$; then $H^{i}\left(L^{N} \otimes B\right)=0$ for $i \neq \frac{1}{2} \operatorname{dim}_{\mathbb{C}} M$. 


\section{VERBITSKY}

Proof. The proof of Theorem 5.8 is similar to the Kodaira-Nakano vanishing for vector bundles of arbitrary rank. The same argument as used in the proof of Theorem 5.6 can be employed to deduce Theorem 5.8 from the following statement.

Proposition 5.9. Let $(M, I, J, K)$ be a compact, irreducible hyperkähler manifold, $L$ a holomorphic line bundle on $(M, I)$ with $c_{1}(L) \neq 0$, and $B$ an arbitrary holomorphic vector bundle on $(M, I)$. Assume that $q\left(c_{1}(L), \omega\right)>0$, where $\omega$ is the Kähler form of $(M, I)$. Then there exists a sufficiently big number $N_{0}$, such that, for any integer $N>N_{0}, H^{i}\left(L^{N} \otimes B\right)=0$ for all $i>\frac{1}{2} \operatorname{dim}_{\mathbb{C}} M$.

Proof. To prove Proposition 5.9, we use the formula (3.4) again:

$$
\Delta_{\bar{\partial}}-\Delta_{\bar{\partial}_{J}}=-\left[\Theta_{+}, \Lambda_{\bar{\Omega}}\right],
$$

where $\Delta_{\bar{\partial}}, \Delta_{\bar{\partial}_{J}}$ are the Laplacians on $L^{N} \otimes B$, and $\Theta_{+}$is the $\Lambda_{+, I}^{1,1}(M) \otimes \operatorname{End}\left(L^{N} \otimes B\right)$ part of the curvature of $L^{N} \otimes B$, considered as an operator on $\Lambda^{(0, *)}(M) \otimes\left(L^{N} \otimes B\right)$ as in the proof of the quaternionic Dolbeault Kodaira-Nakano identity (Theorem 3.6). Since the curvature is additive on tensor product, we have

$$
\Theta_{+}=\Theta_{B}+N \Theta_{L},
$$

where $\Theta_{B}, \Theta_{L}$ are $\Lambda_{+}^{2}(M)$ parts of the curvatures of $B$ and $L$. The same argument as used in the proof of (5.2) implies that $\Theta_{L}=\lambda \bar{\Omega}$, where $\lambda=q\left(c_{1}(L), \omega\right) / q(\omega, \omega)$. Then, as the Lefschetz formula (Proposition 3.1) implies,

$$
-\left[\Theta_{+}, \Lambda_{\bar{\Omega}}\right]=-\left[\Theta_{B}, \Lambda_{\bar{\Omega}}\right]+V,
$$

where $V$ is a scalar operator acting on $(0, i)$-forms as $\lambda(i-n) N, n=\frac{1}{2} \operatorname{dim}_{C} M$. Clearly, $-\left[\Theta_{B}, \Lambda_{\bar{\Omega}}\right]+$ $V$ is positive definite for $N$ sufficiently big and $i>n$. From Remark 5.2 we obtain immediately that ker $\Delta_{\bar{\partial}}=0$ whenever $-\left[\Theta_{B}, \Lambda_{\bar{\Omega}}\right]+V$ is positive definite. This proves Proposition 5.9.

\section{Vanishing of cohomology and nef classes with $q(\eta, \eta)=0$}

\subsection{Nef classes on hyperkähler manifolds}

Definition 6.1. Let $M$ be a Kähler manifold, and $\eta \in H^{1,1}(M)$ a real $(1,1)$-class. Then $\eta$ is called nef (numerically effective) if $\eta$ belongs to a closure $\overline{\mathcal{K}}$ of the Kähler cone $\mathcal{K}$ of $M$. The closure $\overline{\mathcal{K}}$ is called the nef cone. A nef line bundle on $M$ is a line bundle with $c_{1}(L)$ nef; a nef divisor $D$ is one with nef cohomology class.

Consider a compact, irreducible hyperkähler manifold $(M, I, J, K)$. Let $L$ be a holomorphic line bundle on $(M, I)$ which is nef and satisfies

$$
q\left(c_{1}(L), c_{1}(L)\right)=0 .
$$

It was conjectured [GHJ02, Saw03] that $L$ is base point free, that is, defines a holomorphic map

$$
(M, I) \rightarrow \mathbb{P} H^{0}\left(L^{N}\right)
$$

for $N$ sufficiently big. If this is true, then (6.1) is a Lagrangian fibration onto its image [Mat99]. A special case of this conjecture was recently proven by Matsushita [Mat06]. This motivates our interest in the geometry of nef classes satisfying $q(\eta, \eta)=0$.

Proposition 6.2. Let $(M, I, J, K)$ be a compact, irreducible hyperkähler manifold, $\eta \in H_{I}^{1,1}(M, \mathbb{R})$ a non-zero nef class on $(M, I)$, satisfying $q(\eta, \eta)=0$, and $\omega$ a Kähler class on $(M, I)$. Then we have the following:

(i) $q(\omega, \eta)>0$;

(ii) choose a positive real number $\varepsilon<q(\eta, \omega) / q(\omega, \omega)$ then $\eta-\varepsilon \omega$ lies outside of $\overline{\mathcal{K}}^{r} \cup-\overline{\mathcal{K}}^{r}$. 


\section{VANISHING THEOREMS FOR HYPERKÄHLER MANIFOLDS}

Proof. The proof is clear.

\subsection{A vanishing theorem and its applications}

From Proposition 6.2, the following theorem is apparent.

Theorem 6.3. Let $(M, I, J, K)$ be a compact, irreducible hyperkähler manifold, $\operatorname{dim}_{\mathbb{H}} M=n$, and $L$ a non-trivial holomorphic bundle on $(M, I)$ which is nef and satisfies $q\left(c_{1}(L), c_{1}(L)\right)=0$. Consider an ample line bundle $H$ on $(M, I)$. Then there exists $N_{0}$ such that, for all integers $N>N_{0}$,

$$
H^{i}\left(L^{N} \otimes H^{*}\right)=0 \quad \text { for } i \neq n .
$$

Proof. Let $N_{0}=1 / \varepsilon$, where

$$
\varepsilon=\frac{q\left(c_{1}(L), c_{1}(H)\right)}{q\left(c_{1}(H), c_{1}(H)\right)} .
$$

Then $N c_{1}(L)-c_{1}(H) \notin \overline{\mathcal{K}}^{\curvearrowright} \cup-\overline{\mathcal{K}}^{\curvearrowright}$ as follows from Proposition 6.2. The vanishing of (6.2) then follows from Theorem 5.6.

Theorem 6.3 has an immediate corollary.

Corollary 6.4. Let $(M, I, J, K)$ be a compact, irreducible hyperkähler manifold, $\operatorname{dim}_{\mathbb{H}}(M)>1$, $L$ a non-trivial holomorphic bundle on $(M, I)$ which is nef and satisfies $q\left(c_{1}(L), c_{1}(L)\right)=0$, and $D$ an ample divisor on $(M, I)$. Then, for sufficiently big $N>N_{0}$, the natural restriction map

$$
H^{0}\left(L^{N}\right) \rightarrow H^{0}\left(\left.L^{N}\right|_{D}\right)
$$

is surjective.

Proof. The following exact sequence is well known:

$$
\left.0 \rightarrow L^{N}(-D) \rightarrow L^{N} \rightarrow L^{N}\right|_{D} \rightarrow 0 .
$$

By Theorem 5.6, $H^{1}\left(L^{N}(-D)\right)=0$. Then the long exact sequence of cohomology gives

$$
0 \rightarrow H^{0}\left(L^{N}(-D)\right) \rightarrow H^{0}\left(L^{N}\right) \rightarrow H^{0}\left(\left.L^{N}\right|_{D}\right) \rightarrow 0 .
$$

This proves Corollary 6.4.

Corollary 6.4 can be generalized as follows.

Theorem 6.5. Let $(M, I, J, K)$ be an irreducible hyperkähler manifold, and $X \subset(M, I)$ a subvariety of dimension $\operatorname{dim}_{\mathbb{C}} X>\frac{1}{2} \operatorname{dim}_{\mathbb{C}} M$. Assume that $X$ is a complete intersection of ample divisors. Consider a holomorphic line bundle $L$ on $(M, I)$ with $c_{1}(L)$ nef and $q\left(c_{1}(L), c_{1}(L)\right)=0$. Then the natural restriction map is surjective on holomorphic sections:

$$
H^{0}\left(L^{N}\right) \rightarrow H^{0}\left(\left.L^{N}\right|_{X}\right) \rightarrow 0
$$

for a sufficiently big power of $L$.

Proof. The proof follows the standard pattern of how to generalize an assertion for hypersurfaces to one for complete intersections.

\section{ACKNOWLEDGEMENTS}

I am grateful to E. Amerik and D. Markushevich for insightful comments and observations, and to the referee for useful suggestions and corrections. 


\section{VANISHING THEOREMS FOR HYPERKÄHLER MANIFOLDS}

\section{REFERENCES}

Bas92 R. J. Baston, Quaternionic complexes, J. Geom. Phys. 8 (1992), 29-52.

Bea83 A. Beauville, Varietes Kähleriennes dont la première classe de Chern est nulle, J. Differential Geom. 18 (1983), 755-782.

Bes87 A. Besse, Einstein manifolds (Springer, New York, 1987).

Bog74 F. A. Bogomolov, On the decomposition of Kähler manifolds with trivial canonical class, Math. USSR-Sb. 22 (1974), 580-583.

Bog78 F. A. Bogomolov, Hamiltonian Kähler manifolds, Sov. Math. Dokl. 19 (1978), 1462-1465; translation from Dokl. Akad. Nauk SSSR 243 (1978), 1101-1104.

CS88 M. M. Capria and S. M. Salamon, Yang-Mills fields on quaternionic spaces, Nonlinearity 1 (1988), $517-530$.

Fuj85 A. Fujiki, On the de Rham cohomology group of a compact Kähler symplectic manifold, in Algebraic geometry, Sendai, 1985, Advanced Studies in Pure Mathematics, vol. 10 (Mathematical Society of Japan, Tokyo, 1985), 105-165.

GH78 P. Griffiths and J. Harris, Principles of algebraic geometry (Wiley-Interscience, New York, 1978).

GHJ02 M. Gross, D. Huybrechts and D. Joyce, Calabi-Yau manifolds and related geometries, Universitext (Springer, Berlin, 2002).

Hum72 J. Humphreys, Introduction to Lie algebras and representation theory, Graduate Texts in Mathematics (Springer, Berlin, 1972).

LY97 N. C. Leung and S. Yi, Analytic torsion for quaternionic manifolds and related topics, Preprint (1997), dg-ga/9710022.

Mat99 D. Matsushita, On fibre space structures of a projective irreducible symplectic manifold, Topology 38 (1999), 79-83. Addendum, Topology 40 (2001), 431-432.

Mat06 D. Matsushita, On nef reductions of projective irreducible symplectic manifolds, Preprint (2006), math.AG/0601114.

Sal07 S. Salamon, Quaternionic manifolds, Communicazione inviata all'Instituto nazionale di Alta Matematica Francesco Severi.

Saw03 J. Sawon, Abelian fibred holomorphic symplectic manifolds, Turkish J. Math. 27 (2003), 197-230.

Ver90 M. Verbitsky, On the action of the Lie algebra $\mathfrak{s o ( 5 )}$ on the cohomology of a hyperkähler manifold, Funct. Anal. Appl. 24 (1990), 70-71.

Ver95 M. Verbitsky, Cohomology of compact hyperkähler manifolds, Preprint (1995), math.AG/9501001.

Ver96a M. Verbitsky, Hyperholomorphic bundles over a hyperkähler manifold, J. Algebraic Geom. 5 (1996), 633-669.

Ver96b M. Verbitsky, Cohomology of compact hyperkähler manifolds and its applications, Geom. Funct. Anal. 6 (1996), 601-612.

Ver02 M. Verbitsky, Hyperkähler manifolds with torsion, supersymmetry and Hodge theory, Asian J. Math. 6 (2002), 679-712.

Yau78 S. T. Yau, On the Ricci curvature of a compact Kähler manifold and the complex Monge-Ampère equation I, Comm. Pure Appl. Math. 31 (1978), 339-411.

Misha Verbitsky verbit@maths.gla.ac.uk, verbit@mccme.ru

Institute of Theoretical and Experimental Physics, B. Cheremushkinskaya 25, Moscow, 117259, Russia

Current address: University of Glasgow, Department of Mathematics, 15 University Gardens, Glasgow, G12 8QW, UK 